\title{
Experimental investigation of parameters influencing plasmonic nanoparticle-mediated bubble generation with nanosecond laser pulses
}

\author{
Andrew M. Fales \\ William C. Vogt \\ Keith A. Wear \\ T. Joshua Pfefer \\ Ilko K. Ilev
}




\title{
Experimental investigation of parameters influencing plasmonic nanoparticle-mediated bubble generation with nanosecond laser pulses
}

\author{
Andrew M. Fales, ${ }^{*}$ William C. Vogt, Keith A. Wear, T. Joshua Pfefer, and Ilko K. Ilev \\ U.S. Food and Drug Administration, Office of Science and Engineering Laboratories, Center for Devices and Radiological Health, Silver Spring, \\ Maryland, United States
}

\begin{abstract}
Plasmonic nanoparticles (PNPs) continue to see increasing use in biophotonics for a variety of applications, including cancer detection and treatment. Several PNP-based approaches involve the generation of highly transient nanobubbles due to pulsed laser-induced vaporization and cavitation. While much effort has been devoted to elucidating the mechanisms behind bubble generation with spherical gold nano particles, the effects of particle shape on bubble generation thresholds are not well understood, especially in the nanosecond pulse regime. Our study aims to compare the bubble generation thresholds of gold nanospheres, gold nanorods, and silica-core gold nanoshells with different sizes, resonances, and surface coatings. Bubble generation is detected using a multimodality microscopy platform for simultaneous, nanosecond resolution pump-probe imaging, integrated scattering response, and acoustic transient detection. Nanoshells and large (40-nm width) nanorods were found to have the lowest thresholds for bubble generation, and in some cases they generated bubbles at radiant exposures below standard laser safety limits for skin exposure. This has important implications for both safety and performance of techniques employing pulsed lasers and PNPs. $\odot$ The Authors. Published by SPIE under a Creative Commons Attribution 4.0 Unported License. Distribution or reproduction of this work in whole or in part requires full attribution of the original publication, including its DOI. [DOI: 10.1117/1.JBO.24.6.065003]
\end{abstract}

Keywords: plasmonic nanoparticle; nanorod; nanoshell; pulsed laser; nanosecond; nanobubble.

Paper 190075R received Mar. 20, 2019; accepted for publication May 30, 2019; published online Jun. 22, 2019.

\section{Introduction}

Plasmonic nanoparticles (PNPs) have shown great promise for applications in optical diagnostics and therapeutics due to the unique optical properties arising from their surface plasmon resonance (SPR). ${ }^{1}$ By controlling PNP size, shape, and material, the spectral region where the SPR occurs can be tuned. For nanobiophotonic applications, the near-infrared (NIR) region is often of interest due to greater light penetration in tissue. Commonly used PNPs with SPR in the NIR include gold nanorods and nanoshells. The high extinction cross section and tunable spectra of these PNPs may be harnessed for diagnostic techniques, such as surface-enhanced spectroscopies ${ }^{2-4}$ and photoacoustic imaging, ${ }^{5}$ as well as therapeutics, such as selective photothermal and photomechanical ablation. ${ }^{6-8}$

Owing to strong SPR region absorption, PNPs undergoing pulsed irradiation may experience rapid heating and subsequent vaporization of adjacent water molecules, leading to generation of transient bubbles. Cavitation bubble-driven ablation using pulsed lasers and PNPs is an exciting new technique that has been demonstrated to be effective for in vivo cancer detection and treatment, as well as optoporation. ${ }^{8,9}$ PNPs can be delivered to cancerous tissues intravenously using passive uptake through the enhanced permeation and retention effect or active targeting when combined with agents such as antibodies. At relatively low pulse energies, optical exposure of cells/tissue containing PNPs generates "small" bubbles $(<10 \mu \mathrm{m}$ diameter $)$ that may be detected by optical (in vitro) and/or acoustic (in vitro and in vivo) techniques without causing any adverse effects to the

*Address all correspondence to Andrew M. Fales, E-mail: andrew.fales@ fda.hhs.gov cells/tissues. ${ }^{10}$ Once a cancerous area has been located, therapeutic levels of laser energy can be delivered to create larger bubbles ( $>10 \mu \mathrm{m}$ diameter) that mechanically disrupt the diseased cells. ${ }^{8,10}$ The advantage of using pulsed lasers capable of producing mechanical damage as opposed to longer pulses that lead to greater heating, is that the damaged area is highly localized to cancerous cells that have bound/uptaken the targeted gold nanoparticles, allowing normal cells in the laser-irradiated area to survive. In contrast, photothermal therapy with continuous wave or longer pulsed lasers has the potential to collaterally damage healthy tissues around the tumor.

Thresholds for bubble generation with gold nanospheres and nanosecond pulses reported in the literature vary greatly. Theoretical models predict thresholds below the ANSI maximum permissible exposure (MPE) for skin $\left(20 \mathrm{~mJ} / \mathrm{cm}^{2}\right.$ at $532 \mathrm{~nm}$ ), while experimental values typically range between 100 and $1000 \mathrm{~mJ} / \mathrm{cm}^{2}$ at this wavelength. ${ }^{1-15}$ Two studies investigating the effect of particle size on bubble generation threshold experimentally had an order of magnitude difference in determined thresholds for similarly sized particles. ${ }^{11,12}$ There have been limited reports on the use of other PNP shapes for bubble generation, and many have been performed using femtosecond pulsed lasers. ${ }^{16-22}$ With diagnostic biophotonic techniques employing NIR-resonant nanoparticles and nanosecond pulsed lasers, such as photoacoustic imaging, ${ }^{23-25}$ it is important to evaluate the possibility of bubble generation, which may lead to unintended biological effects.

Owing to the wide variation of nanosphere bubble thresholds reported in the literature, and lack of information about the effect of nanoparticle shape, we have decided to experimentally investigate several factors that may impact PNP-mediated bubble generation with nanosecond laser pulses. These findings will 
provide greater insight into the safety and performance of diagnostic and therapeutic products combining PNPs and nanosecond pulsed lasers. The goal of this study is to evaluate experimentally the effects of PNP size, shape, resonance, and surface properties on bubble generation thresholds. We first use different sizes of gold nanospheres to validate test methods for quantifying bubble generation thresholds and compare our findings with previously reported values. We then measure the bubble generation thresholds of nanorods and silica-core gold nanoshells and quantify the effect of varying resonance peak/size, and surface coating.

\section{Methods}

\subsection{Nanoparticle Characterization}

Gold nanospheres (20-, 40-, 60-, 80-, 100-nm diameter) with resonance peaks near $532 \mathrm{~nm}$ were purchased from Cytodiagnostics (Burlington, Ontario, Canada). Gold nanorods with the same resonance peak $(850 \mathrm{~nm})$ and different diameters $(10,25,40 \mathrm{~nm})$ were obtained from Nanopartz Inc. (Loveland, Colorado). The actual nanorod dimensions were as follows: 45nm length, 10-nm width, 4.5 aspect ratio (AR); 93-nm length, 25-nm width, 3.7 AR; 160-nm length, 44-nm width, 3.6 AR. NanoHybrids Inc. (Austin, Texas) supplied polyethylene glycol (PEG)-coated and silica-coated gold nanorods with 850-nm resonance. Gold nanorods with citrate and PEG surfaces at $800-\mathrm{nm}$ resonance, gold nanoshells with different resonances $(660,800,980 \mathrm{~nm})$ and surface coatings [polyvinylpyrrolidone (PVP), lipoic acid (LA), and PEG] were procured from nanoComposix (San Diego, California). The 660-nm resonant nanoshells are $158 \mathrm{~nm}$ in diameter, with a silica core of 88- and 35-nm-thick gold shell. The 800-nm resonant nanoshells have a diameter of $154 \mathrm{~nm}$, with a 120-nm core and 17-nm shell. The 980-nm resonant nanoshells are $230 \mathrm{~nm}$ in diameter with a core of 198- and 16-nm shell. Transmission electron microscopy (TEM) micrographs were recorded on a JEM1400 (JEOL, Inc.; Peabody, Massachusetts) operating at an accelerating voltage of $80 \mathrm{kV}$. Samples for TEM were prepared by drop-casting $7 \mu \mathrm{L}$ of nanoparticle solution onto a Formvar- carbon-coated copper grid (FCF-400Cu, Electron Microscopy Sciences; Hatfield, Pennsylvania) and allowing it to dry. Optical extinction spectra were collected using a dual-beam UV/VIS/NIR spectrophotometer (Lambda 1050, PerkinElmer; Waltham, Massachusetts).

\subsection{Multimodal Microscopy Setup}

Nanobubble generation and detection were performed on a multifunctional microscopy platform incorporating pump-probe imaging, integrated scattering response, and acoustic detection (Fig. 1). For nanospheres, bubbles were generated by a pump pulse from a frequency-doubled, Q-switched Nd:YAG laser (532-nm wavelength, 5-ns pulse duration, Surelite I-10 with SSP-2, Continuum; San Jose, California) directed into the backport of an inverted microscope (IX71, Olympus; Center Valley, Pennsylvania). To excite nanorods and nanoshells with NIR absorption peaks, an optical parametric oscillator pumped with a third harmonic Nd:YAG (355-nm pump, 5-ns pulse duration, Surelite OPO Plus with SL III-10, Continuum) was used to deliver pump pulses, tuned to the nominal resonance peak wavelength of each sample over a range of radiant exposures. Pumpprobe images were acquired on a CMOS camera (DCC3240M, Thorlabs; Newton, New Jersey) using a delay generator (Stanford Research Systems DG535; Sunnyvale, California) to precisely adjust the timing of a 575-nm-wavelength probe pulse from a nitrogen dye laser (MNL 205, Rhodamine 6G laser dye, Lasertechnik Berlin; Berlin, Germany) that illuminated the sample from above at a 45-deg angle. The integral scattering response of the generated bubbles was monitored by measuring the transmitted intensity of a 632.8-nm wavelength $\mathrm{He}-\mathrm{Ne}$ laser (Model 117A, Spectra-Physics; Santa Clara, California) through the sample with a photodiode (APD110A, Thorlabs) on a digital oscilloscope (TDS2024C, Tektronix; Beaverton, Oregon). Acoustic transients were detected with a miniature $10 \mathrm{MHz}, 2-\mathrm{mm}$ diameter planar immersion ultrasonic transducer (XMS-310, Olympus IMS; Waltham, Massachusetts) placed in the sample chamber, using a digital oscilloscope (TDS2014, Tektronix) after $40 \mathrm{~dB}$ preamplification (5678 preamplifier, Olympus IMS).

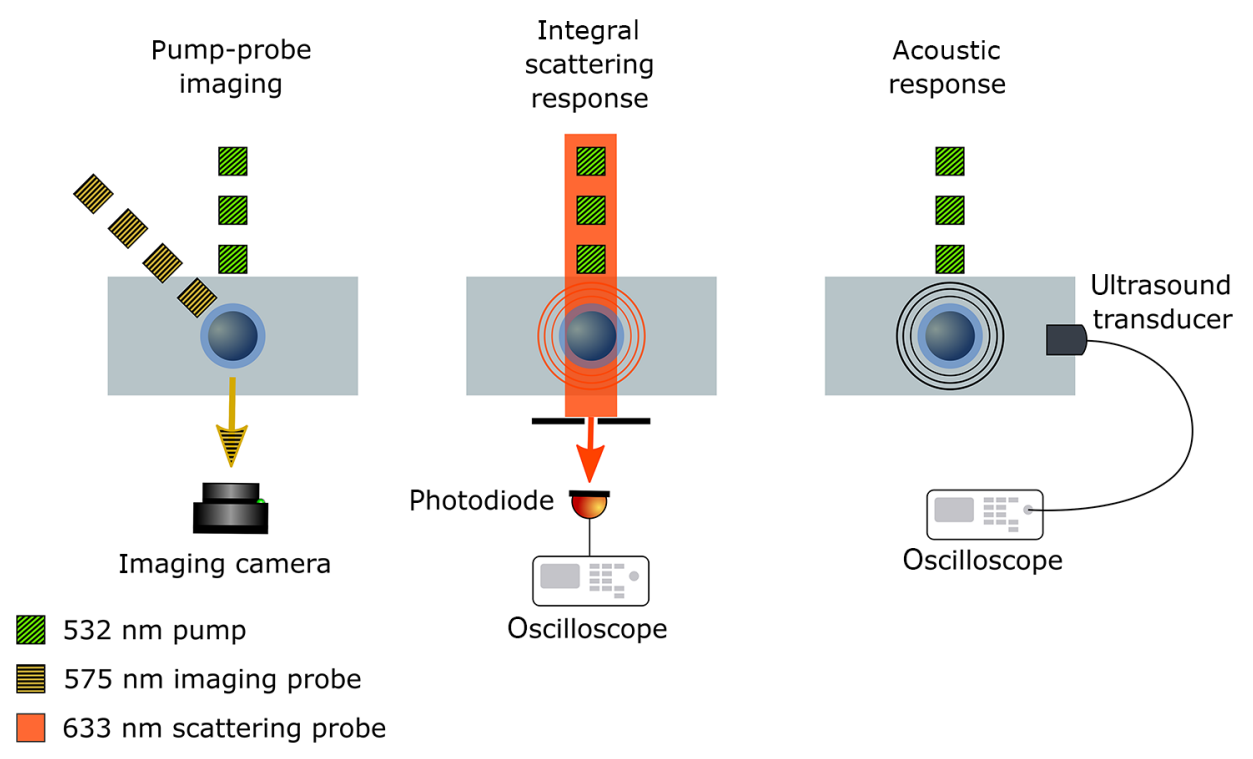

Fig. 1 Schematic representation of the microscope setup. 
Table 1 MPE values for skin at $10 \mathrm{~Hz}$ pulse repetition rate over various wavelengths used in this study.

\begin{tabular}{lcccccc} 
Wavelength $(\mathrm{nm})$ & $400-700$ & 750 & 800 & 850 & 950 & 980 \\
\hline MPE $\left(\mathrm{mJ} / \mathrm{cm}^{2}\right)$ & 20 & 25.2 & 31.7 & 39.9 & 63.2 & 72.6 \\
\hline
\end{tabular}

\subsection{Data Analysis and Simulations}

Data analysis was performed using Python 2.7 with the Anaconda Python distribution. Absorption cross sections of nanospheres and nanoshells were calculated from Mie theory with MiePlot v4.6. Absorption cross sections of nanorods were computed using the discrete dipole approximation code ADDA 1.3b4. ${ }^{26}$ Bubble generation thresholds were determined using all three modalities on the microscope setup. Fifty pulses were used to irradiate each sample over a range of radiant exposures and the bubble response was recorded. Any pump-probe bubble image was recorded as a positive bubble response. If no bubbles were seen in the image but a scattering and acoustic response were both observed, this was also considered as a positive response. These binary data were plotted against their corresponding radiant exposures, and a logistic regression was performed. Owing to the stochastic nature of the bubble generation process, bubble threshold was defined as the $50 \%$ probability of bubble formation determined from logistic regression. ${ }^{27}$

A reference table for the per-pulse ANSI MPEs for skin exposure at wavelengths used in this study is shown in Table $1 .{ }^{15}$

\section{Results and Discussion}

\subsection{Nanosphere Bubble Thresholds}

To study the bubble generation phenomena associated with pulsed laser-nanoparticle interaction, we built a multimodality microscopy platform for pump-probe imaging, integrated scattering response, and acoustic response detection (Fig. 1). Each of the three detection methods has its own advantages and disadvantages. Pump-probe imaging is the most sensitive technique, allowing observation of any bubbles generated within the focal plane of the objective, although bubbles outside of the depth of focus or smaller than the optical diffraction limit will not be detected. Since a specific pump-probe delay must be selected to capture an image, this method cannot provide information about the bubble lifetime. The integrated scattering response provides a method for estimating bubble lifetime, as bubble formation and collapse scatter the $\mathrm{He}-\mathrm{Ne}$ probe beam and produce a decrease in sample transmittance. Measured transmittance is affected by any bubble generated within the probe beam path, not just those within the beam focus. The disadvantage of this technique is that it has low sensitivity and may measure the response due to multiple bubbles even though only a single bubble is imaged. Acoustic transient detection is advantageous for detection of bubbles in turbid media, where the pump-probe and scattering response would be unusable, but the shortest detectable bubble lifetime is restricted by the acoustic transducer impulse response, which has a duration inversely proportional to the transducer bandwidth, that may be longer than a bubble lifetime. Therefore, the duration of the acoustic transient measurement only provides an upper bound for the bubble lifetime. Another disadvantage of the acoustic response detection is that acoustic transients due to thermoelastic expansion and cavitation have different amplitudes but similarly shaped waveforms, making it difficult to determine whether bubbles are being generated based on the acoustic signal alone.

Examples of the data obtained with the different modalities are shown in Fig. 2. A submicron bubble appears as a bright dot in the pump-probe image [Fig. 2(a)]. The integral scattering response [Fig. 2(b)] has a duration of $\sim 150 \mathrm{ns,} \mathrm{which} \mathrm{corre-}$ sponds to the bubble lifetime. The acoustic response of the generated bubble is shown in Fig. 2(c), starting at $~ 350 \mathrm{~ns}$. The secondary waveform observed around $800 \mathrm{~ns}$ is due to the reflection off the wall of the sample chamber. Note that the time scales in Figs. 2(b) and 2(c) are not absolute as the oscilloscopes were triggered independently.

TEM micrographs of different diameter nanospheres used in this study are shown in Fig. 3(a). Figure 3(b) shows an example of the probability curves that were used to determine bubble thresholds. As seen in Fig. 3(c) and Table 2, there is a large decrease in bubble threshold as nanosphere diameter increases from 20 to $60 \mathrm{~nm}$, and a much slower change from 60 to $100 \mathrm{~nm}$. The experimentally determined bubble thresholds scale inversely with their absorption cross sections $\left(C_{\mathrm{abs}}\right)$. The shape of this curve is similar to what has been reported in the literature, although there is still debate as to whether there should be a local minimum at $60 \mathrm{~nm}$ (above this size the absorption cross section is no longer proportional to the particle volume). ${ }^{11,12,14,19,28}$ These results also follow the same trend with particle size that we have observed for nanosphere damage thresholds. $^{29}$
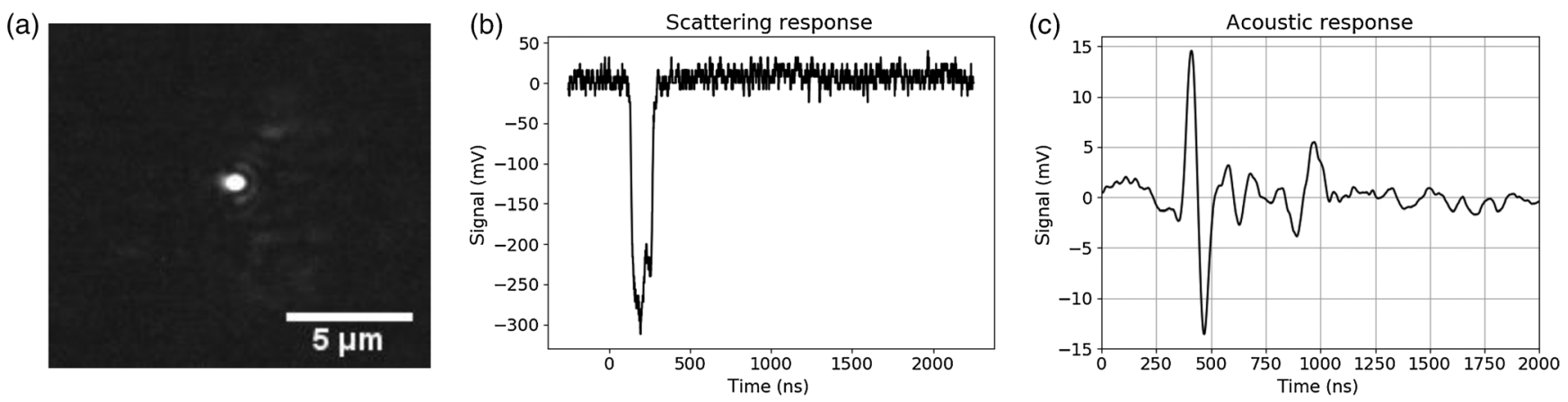

Fig. 2 (a) Representative pump-probe image, (b) integral scattering response, and (c) acoustic response of a nanobubble. 
(a)

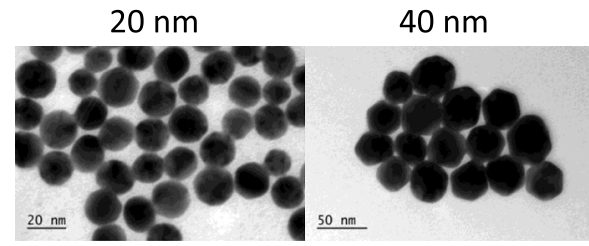

\section{Nanosphere diameter}
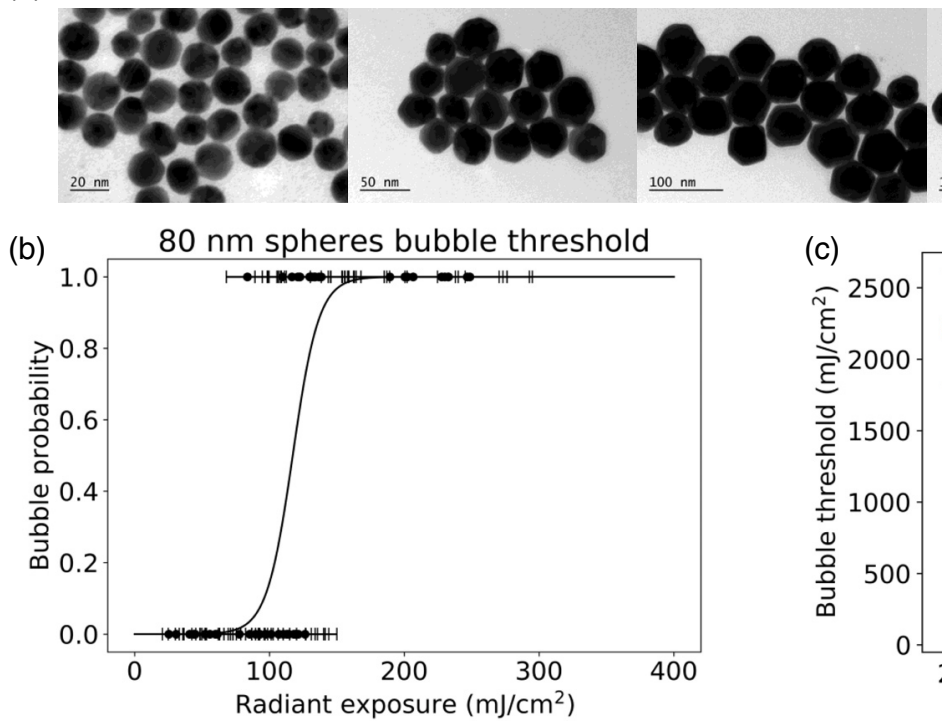

$80 \mathrm{~nm}$ $100 \mathrm{~nm}$
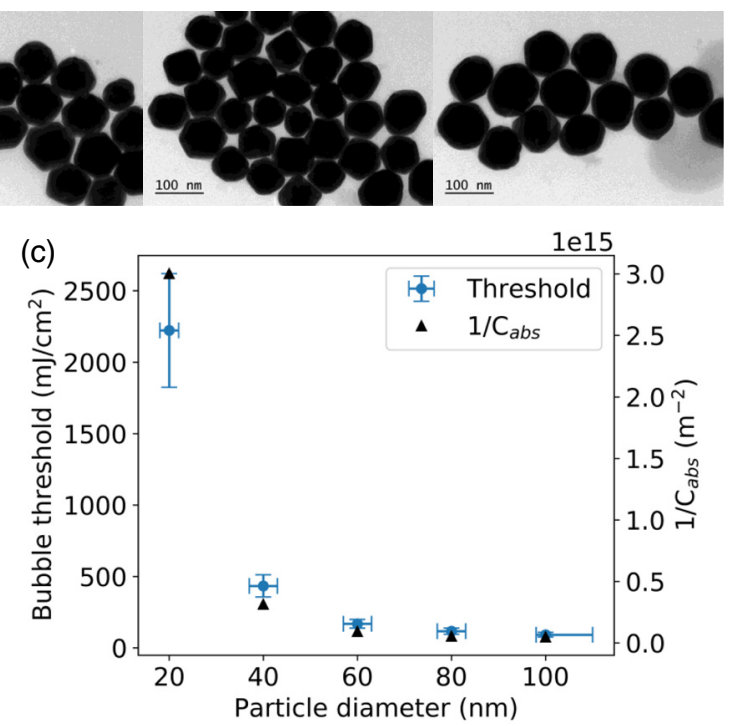

Fig. 3 (a) Representative TEM micrographs of the nanosphere samples. (b) Example of the bubble generation probability curves that are used to determine the bubble thresholds. (c) Bubble generation thresholds as a function of particle diameter (circles, left axis) and their corresponding inverse absorption cross sections (triangles, right axis).

Table 2 Tabulated nanosphere bubble thresholds.

\section{Particle}

\begin{tabular}{lccccc} 
diameter $(\mathrm{nm})$ & 20 & 40 & 60 & 80 & 100 \\
\hline $\begin{array}{l}\text { Bubble } \\
\text { threshold } \\
\left(\mathrm{mJ} / \mathrm{cm}^{2}\right)\end{array}$ & $2200 \pm 400$ & $430 \pm 80$ & $170 \pm 30$ & $120 \pm 20$ & $90 \pm 20$ \\
\hline
\end{tabular}

\subsection{Nanoshell Bubble Thresholds}

\subsubsection{Effect of resonance peak}

TEM micrographs of the nanoshells are shown in Fig. 4(a). All three samples were found to have similar bubble generation thresholds [Fig. 4(b) and Table 3] when excited at their plasmon peak. A previous report by Ogunyankin et al. ${ }^{22}$ using hollow

(a)

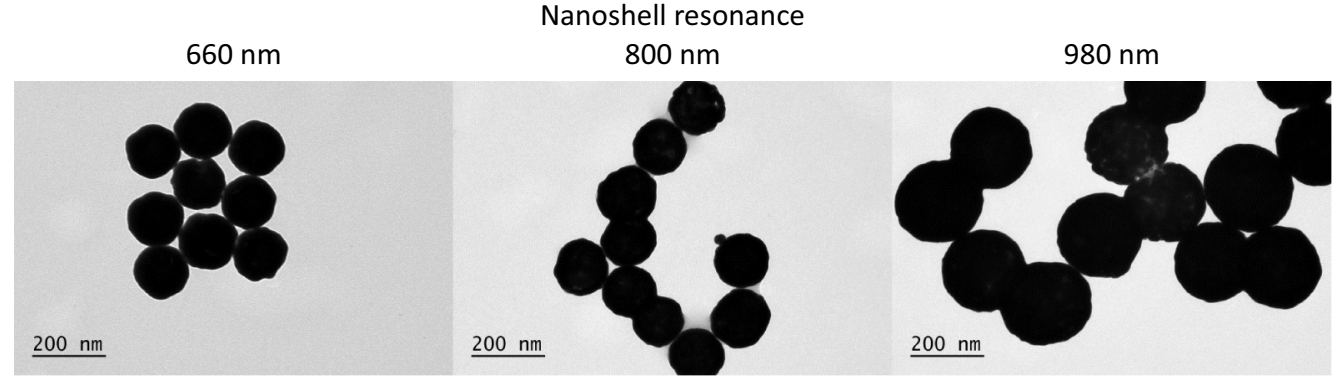

(b)

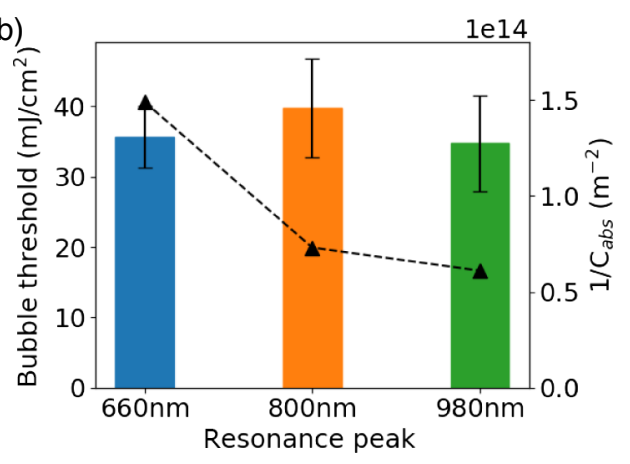

(c)

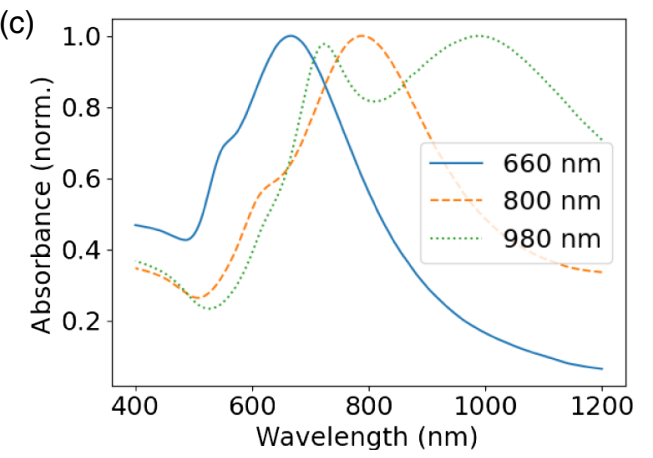

Fig. 4 (a) TEM micrographs of the nanoshells with different resonance peaks. (b) Bubble generation thresholds for the three different nanoshells (bars, left axis) and the inverse of their calculated absorption cross sections (triangles, right axis). Error bars denote standard deviations. (c) Normalized absorbance spectra of the nanoshell samples. 
Table 3 Bubble thresholds for nanoshells with different resonance peaks.

\begin{tabular}{lccc} 
Nanoshell resonance $(\mathrm{nm})$ & 660 & 800 & 980 \\
\hline Bubble threshold $\left(\mathrm{mJ} / \mathrm{cm}^{2}\right)$ & $36 \pm 4$ & $40 \pm 7$ & $35 \pm 7$ \\
\hline
\end{tabular}

gold nanoshells with picosecond laser pulses noted similar behavior. The fact that the bubble thresholds do not scale with the inverse of their $C_{\text {abs }}$ suggests that other factors, such as shell thickness or overall particle size, influence the bubble generation process. While all three bubble generation thresholds were similar in magnitude, the radiant exposures required for 660- and 800-nm resonant shells $\left(36 \pm 4\right.$ and $40 \pm 7 \mathrm{~mJ} / \mathrm{cm}^{2}$; Table 3) were higher than the ANSI MPE for skin at those wavelengths (20 and $31.7 \mathrm{~mJ} / \mathrm{cm}^{2}$; Table 1). The $980-\mathrm{nm}$ resonant shells were found to have a threshold value $\left(35 \pm 7 \mathrm{~mJ} / \mathrm{cm}^{2}\right.$; Table 3) of about half the MPE $\left(72.6 \mathrm{~mJ} / \mathrm{cm}^{2}\right.$; Table 1). It is important to note that in the case of the 660- and 800-nm resonant shells, scattering in tissue may produce fluences that reach the determined threshold levels even when the incident radiant exposure is at or below the MPE. ${ }^{30}$ This indicates that there is a significant potential for bubble generation when using gold nanoshells combined with diagnostic-level pulsed laser irradiation in vivo.

\subsubsection{Effect of surface modification}

A variety of surface modifications can be used to impart nanoparticles with different physical and chemical properties. There is currently limited information on whether these modifications have any impact on plasmonic nanobubble generation. In the case of thiolated surface modifications, such as lipoic acid and PEG, they are known to detach from the particle surface under
Table 4 Bubble thresholds for nanoshells with different surface modifications.

\begin{tabular}{lccc} 
Nanoshell surface & PVP & LA & PEG \\
\hline Bubble threshold $\left(\mathrm{mJ} / \mathrm{cm}^{2}\right)$ & $40 \pm 7$ & $34 \pm 6$ & $39 \pm 7$ \\
\hline
\end{tabular}

laser exposure and are not expected to impact the bubble generation process. ${ }^{31}$ We have compared bubble thresholds for $800-\mathrm{nm}$ resonant nanoshells modified with a physisorbed polymer (PVP), and monodentate (PEG), and bidentate (LA) thiols [Fig 5 and Table 4]. As shown, similar thresholds were found, regardless of the surface modification, which is again in agreement with Ogunyankin et al., ${ }^{22}$ who found similar thresholds for both citrate and PEG-modified hollow nanoshells.

\subsection{Nanorod Bubble Thresholds}

\subsubsection{Effect of nanorod size}

Gold nanorods are commonly used with a number of different optical techniques due to the wide tuning range of their plasmon resonance. One interesting feature of nanorods is that different-sized particles can have the same resonance peak by tuning the $\mathrm{AR} .{ }^{32}$ To study the effect of nanorod size on bubble thresholds, we used nanorods with widths of 10,25 , and $40 \mathrm{~nm}$, all with ARs resulting in plasmon peaks near $850 \mathrm{~nm}$. The effective radii of these nanorods (radius of a volume equivalent sphere) are almost equivalent to their denoted widths. Bubble generation threshold decreased with increasing particle size, and roughly scaled with the inverse of $C_{\mathrm{abs}}$ [Fig. 6, Table 5]. Nanorod damage thresholds have been shown to display the same trend, which is attributed to less-efficient heat transfer to the medium as the surface-to-volume ratio

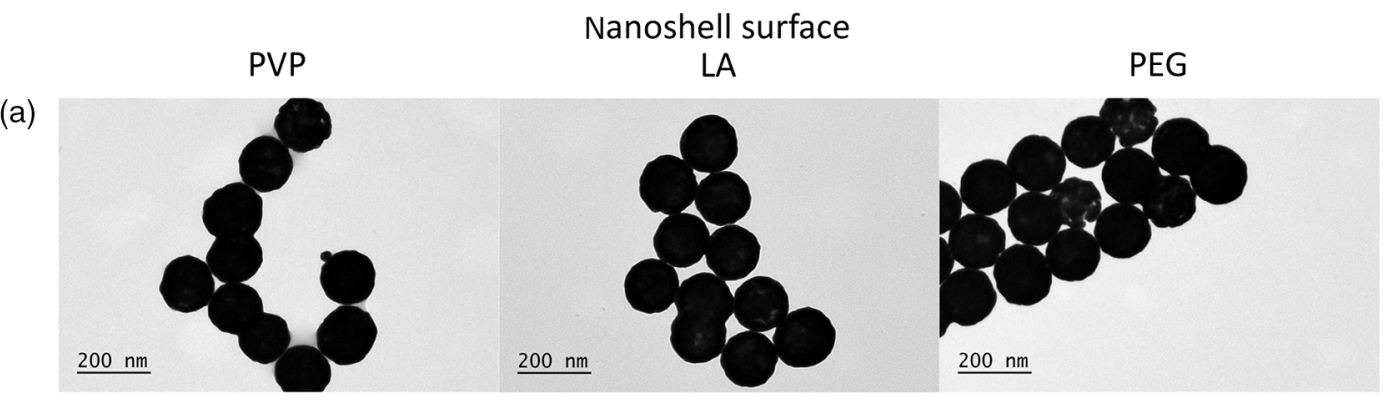

(b)

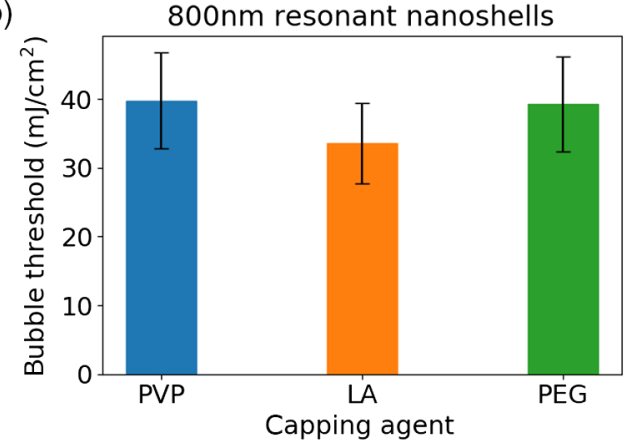

(c)

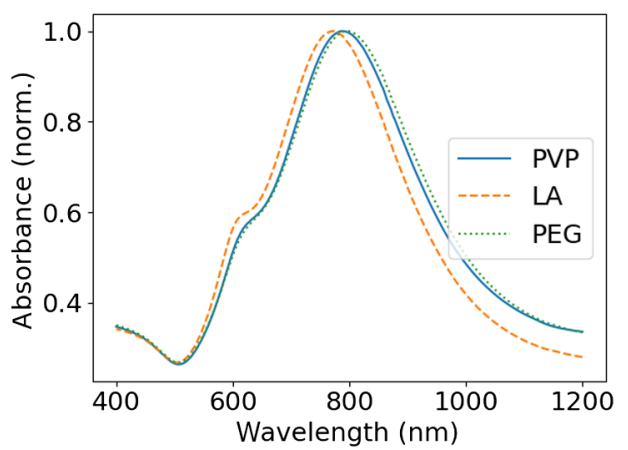

Fig. 5 (a) TEM micrographs of the nanoshells with different surface modifications. (b) Bubble generation thresholds for the three different nanoshells. Error bars denote standard deviation. (c) Normalized absorbance spectra of the nanoshell samples. 


\section{Nanorod width}

(a)

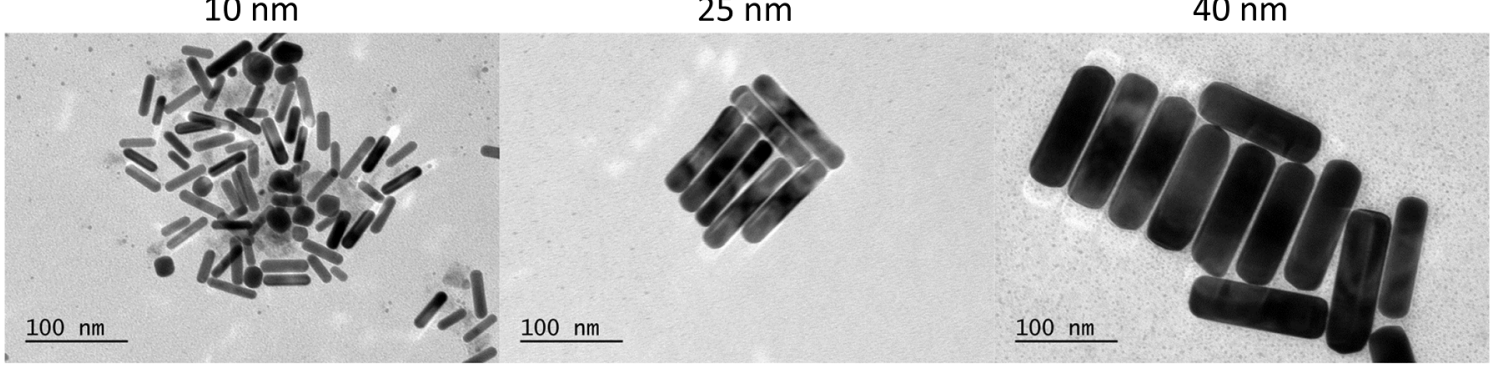

(b)

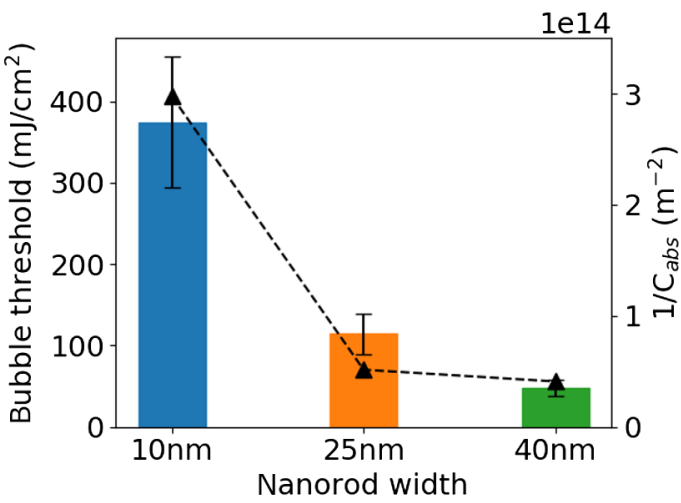

(c)

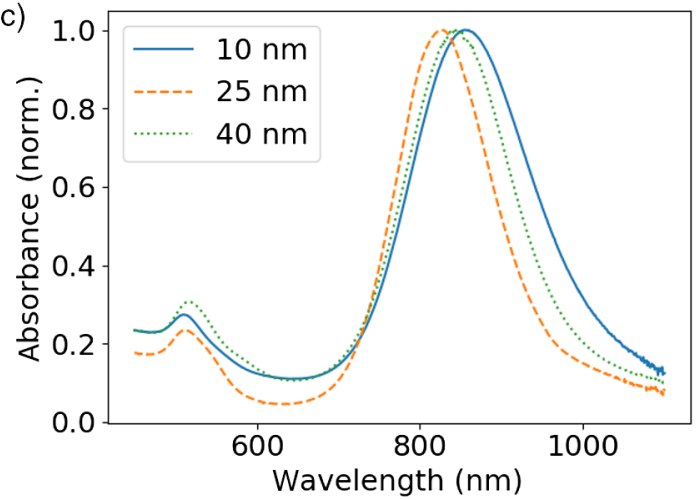

Fig. 6 (a) TEM micrographs of the nanorods with different surface modifications. (b) Bubble generation thresholds for the three different nanorods (bars, left axis) and the inverse of their calculated absorption cross sections (triangles, right axis). Error bars denote standard deviation. (c) Absorbance spectra of the nanorod samples.

Table 5 Bubble thresholds for nanorods with different widths at the same resonance peak.

\begin{tabular}{lccc} 
Nanorod width $(\mathrm{nm})$ & 10 & 25 & 40 \\
\hline Bubble threshold $\left(\mathrm{mJ} / \mathrm{cm}^{2}\right)$ & $350 \pm 80$ & $120 \pm 30$ & $43 \pm 9$ \\
\hline
\end{tabular}

decreases. ${ }^{33}$ For the smallest nanorod size (10-nm width), the bubble generation threshold $\left(350 \mathrm{~mJ} / \mathrm{cm}^{2}\right.$; Table 5) was about an order of magnitude higher than those obtained for nanoshells (34 to $40 \mathrm{~mJ} / \mathrm{cm}^{2}$; Table 3), whereas the $40-\mathrm{nm}$ width nanorods had a comparable threshold $\left(43 \mathrm{~mJ} / \mathrm{cm}^{2}\right.$; Table 5$)$. This indicates that smaller nanorods are less efficient for bubble generation due to faster heat dissipation in the surrounding medium.

\subsubsection{Effect of surface modifications and coatings}

The effect of surface modification on nanorod bubble thresholds was studied using citrate and PEG functionalized nanorods with an 800-nm resonance peak (Fig. 7). As seen in Fig. 7(b), there was no significant difference in the determined bubble thresholds with citrate $\left(630 \pm 90 \mathrm{~mJ} / \mathrm{cm}^{2}\right)$ or PEG $\left(750 \pm 110 \mathrm{~mJ} / \mathrm{cm}^{2}\right)$ surfaces. This was expected due to the detachment of surface ligands from the nanorod surface under laser irradiation.

Silica $\left(\mathrm{SiO}_{2}\right)$ coating has been used to improve nanorod stability for photoacoustic imaging and has also resulted in a threefold increase in photoacoustic intensity. ${ }^{34}$ The proposed mechanism for this enhancement is improved heat transfer to the surrounding medium - where the pressure wave originatesdue to decreased thermal interface resistance. Since more rapid heating of water should impact the onset of vaporization, we evaluated the effect of silica coating on nanorod bubble formation thresholds. A comparison of results obtained during irradiation of gold nanorods with PEG and silica coatings is shown in Fig. 8. The silica-coated particles were found to have a significantly lower bubble threshold $\left(190 \pm 40 \mathrm{~mJ} / \mathrm{cm}^{2}\right)$ than the PEGylated ones $\left(750 \pm 160 \mathrm{~mJ} / \mathrm{cm}^{2}\right)$. Although the silica coating produced a notable reduction in the bubble generation threshold, the radiant exposure required still exceeds the MPE by a factor of 5 .

\subsection{Theoretical Analysis of Nanoparticle Heating}

While gold nanospheres showed good agreement between their $C_{\text {abs }}$ and bubble thresholds, the nanoshells and nanorods exhibited notable discrepancies. The nanorods with widths of 25 and $40 \mathrm{~nm}$ have similar $C_{\text {abs }}$ but $\sim 3 \times$ difference in bubble thresholds. This may be due to differences in the thermal relaxation times between the sizes of nanorods. Using the spherical volume-equivalent effective radii of these two nanorods, their thermal relaxation times were calculated according to Pustovalov et al.

$\tau_{T} \sim \frac{r_{0}^{2} c_{0} \rho_{0}}{3 k_{\infty}}$

where $r_{0}$ is the effective radius, $c_{0}$ and $\rho_{0}$ are the heat capacity and density of gold, and $k_{\infty}$ is the thermal conductivity of water. ${ }^{35}$ This resulted in a value of $1.6 \mathrm{~ns}$ for the $40-\mathrm{nm}$ rods and $0.51 \mathrm{~ns}$ for the $25-\mathrm{nm}$ rods. Thermal confinement is not achieved for either size with a 5-ns laser pulse width. This $\sim 3 \times$ difference in thermal relaxation rates could explain the 
(a)

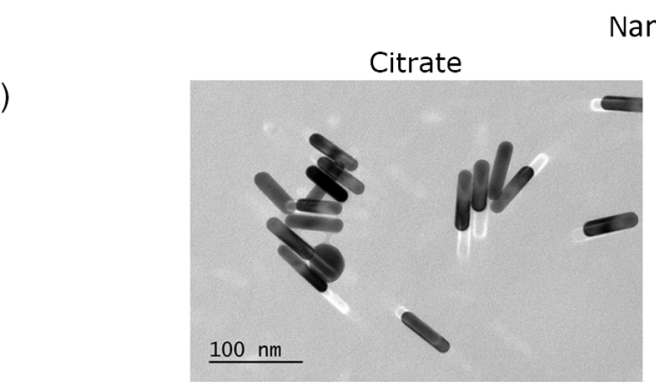

Nanorod surface

(b)

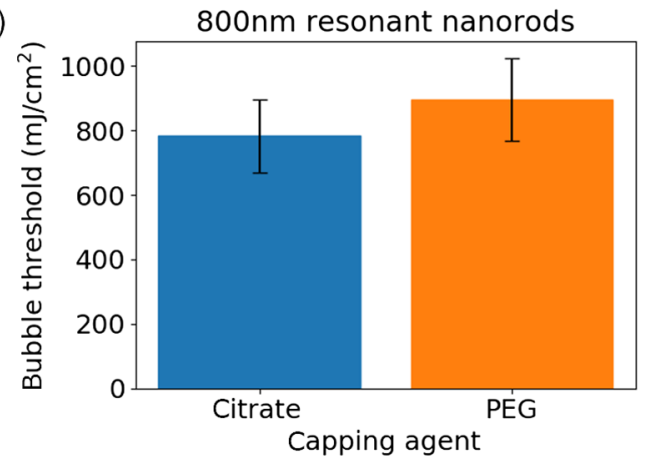

PEG
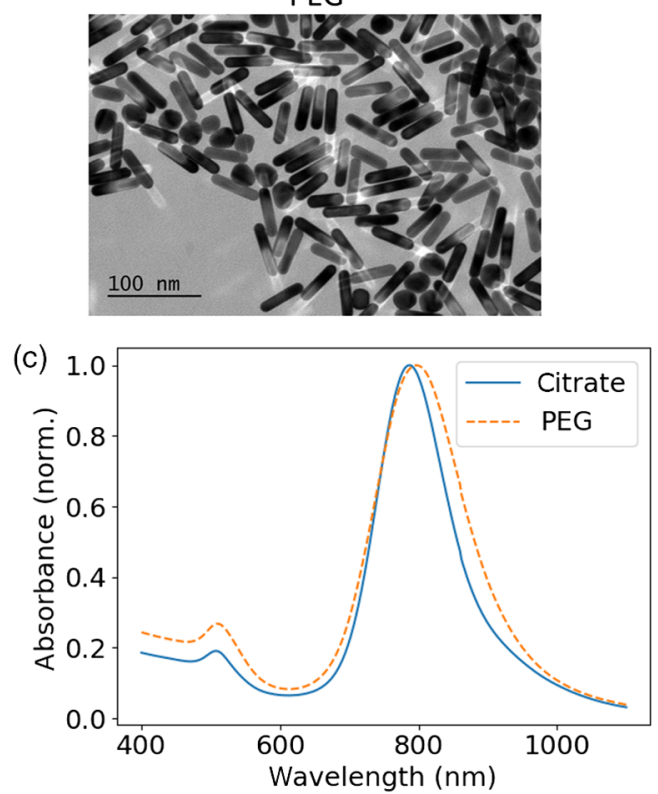

Fig. 7 (a) TEM micrographs of the nanorods with different surface modifications. (b) Bubble generation thresholds for the two nanorods. Error bars denote standard deviation. (c) Absorbance spectra of the nanorod samples.

(a)

PEG

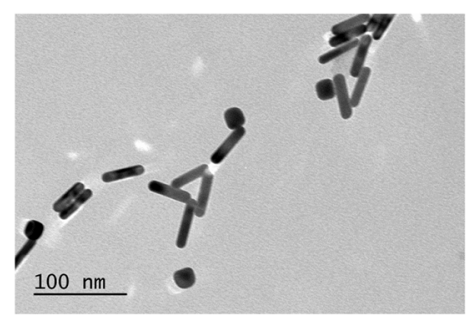

(b)

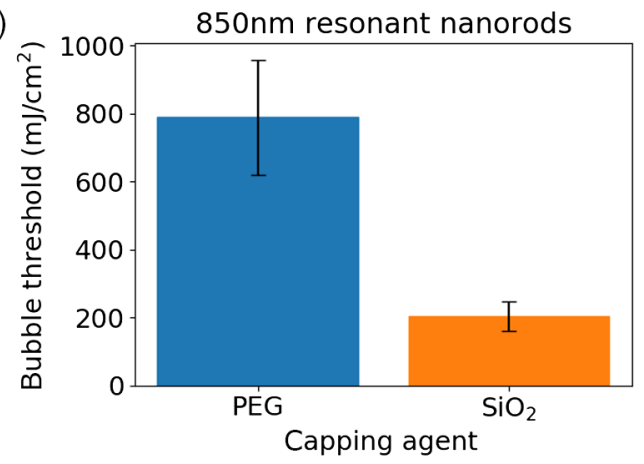

Nanorod coating

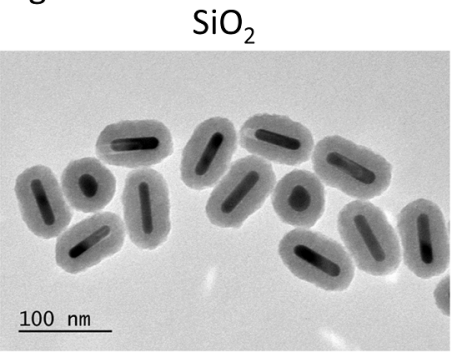

(c)

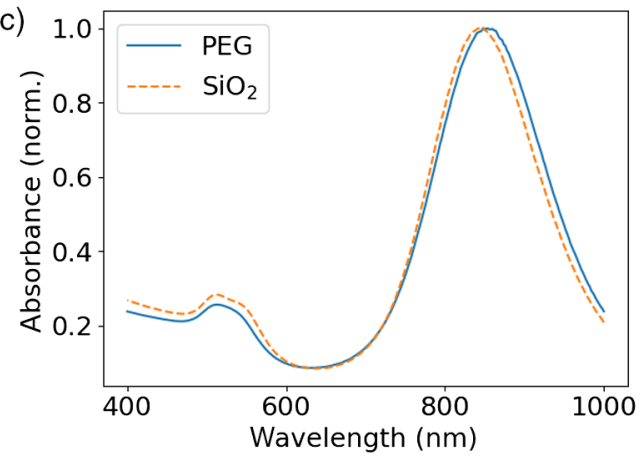

Fig. 8 (a) TEM micrographs of the nanorods with and without silica coating. (b) Bubble generation thresholds for the two nanorods. Error bars denote standard deviation. (c) Absorbance spectra of the nanorod samples.

differences in the observed bubble thresholds, which are of a similar magnitude.

In the case of the nanoshells, the thermal relaxation times were calculated as

$\tau_{T 1}=\frac{r_{0}^{2} c_{0} \rho_{0}}{3 k_{\infty}} \frac{r_{0}}{r_{1}}\left[1+\frac{c_{1} \rho_{1}}{c_{0} \rho_{0}}\left(\frac{r_{1}^{3}}{r_{0}^{3}}-1\right)\right]$, where the subscript 1 refers to properties of the shell and subscript 0 refers to properties of the core. ${ }^{35}$ The 660,800 , and $980 \mathrm{~nm}$ nanoshells had thermal relaxation times of 8.3, 7.2, and $15.4 \mathrm{~ns}$, respectively. These differences in thermal relaxation times alone do not account for the discrepancy between the trend in $C_{\text {abs }}$ and experimental bubble thresholds. Since the thermal relaxation times are on the order of magnitude of 


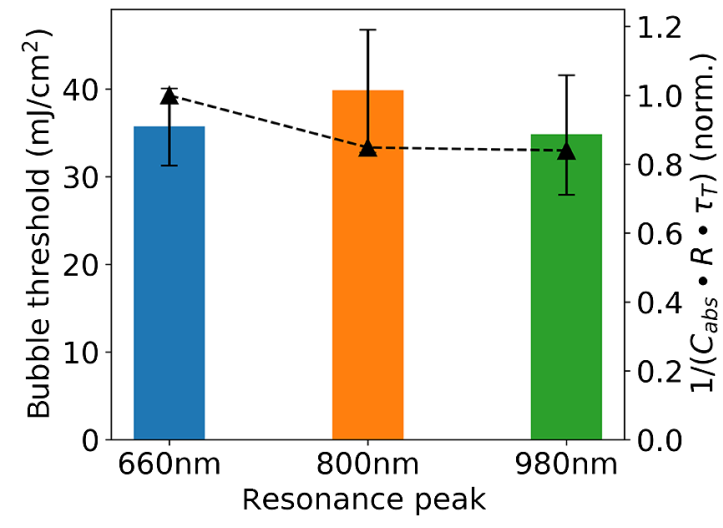

Fig. 9 Nanoshell bubble thresholds (bars, left axis) compared with the inverse of their $C_{\text {abs }}$ after normalization to the thermal relaxation time and resistivity (triangles, right axis).

the laser pulse width, thermal interface resistance may impact the temperatures reached in the nanoshells. The total thermal resistance of each nanoshell was calculated as

$$
\begin{aligned}
R_{\mathrm{tot}} & =R_{0}+R_{\text {shell }}+R_{1} \\
& =\frac{1}{4 \pi r_{0}^{2} g_{0}}+\frac{r_{1}-r_{0}}{4 \pi r_{0} r_{1} k_{\text {gold }}}+\frac{1}{4 \pi r_{1}^{2} g_{1}},
\end{aligned}
$$

where $k_{\text {gold }}\left(314 \mathrm{~W} \mathrm{~m}^{-1} \mathrm{~K}^{-1}\right)$ is the thermal conductivity of gold, and $g_{0}$ and $g_{1}$ are the thermal interface conductance of gold to silica and gold to water. In this calculation, the assumption $g_{0}=g_{1}=150 \mathrm{MW} \mathrm{m}^{-2} \mathrm{~K}^{-1}$ was used. ${ }^{36}$ The 660,800 , and $980 \mathrm{~nm}$ shells were found to have thermal resistivities of $5.15 \times 10^{5}, 3.39 \times 10^{5}$, and $1.35 \times 10^{5} \mathrm{~K} / \mathrm{W}$. We then applied the calculated thermal relaxation time and resistance of each nanoshell as scaling factors to their $C_{\text {abs }}$.

As shown in Fig. 9, the inverse of the normalized $C_{\text {abs }}$ now only varies by $\sim 15 \%$ between the three nanoshells, similar to the difference in bubble thresholds observed experimentally. Although the trend in the scaled $C_{\text {abs }}$ and bubble thresholds are not the same, the relative magnitude of these variations falls within the range of experimental uncertainty.

\subsection{Summary of Findings}

Gold nanosphere bubble thresholds were found to be in the same range of radiant exposures as other experimental reports, validating our experimental approach. ${ }^{11,28,37}$ The change in bubble threshold with nanosphere size is inversely correlated with the absorption cross section of the particles and suggests a thermomediated mechanism of bubble generation. All nanosphere bubble thresholds exceeded the MPE; therefore, the use of nanospheres with nanosecond lasers at current exposure limits is not expected to produce bubble generation. However, clustering of nanoparticles when applied in vitro or in vivo, which is known to reduce the bubble generation threshold, may allow for the production of bubbles below the MPE. ${ }^{11}$ This effect is currently under investigation.

Gold nanoshells had the lowest bubble generation thresholds of the three particle shapes that were studied. In the case of 980-nm resonant shells, this threshold was about half the MPE for skin, indicating that bubble generation is a real possibility at "tissue-safe" exposures as would be used in diagnostic applications. Different surface coatings did not affect the bubble thresholds, which is to be expected due to detachment of surface ligands caused by pulsed laser exposure. ${ }^{38}$ No correlation between the nanoshell absorption cross sections and bubble thresholds was observed, suggesting that other physical properties, such as the overall particle size or thickness or the gold shell, have a significant impact on the bubble formation process. The threshold radiant exposure levels for nanoshell bubble generation may also fall below particle damage thresholds. Nanoshells can be specifically designed for bubble generation below the damage threshold when exposed to off-resonant femtosecond pulses, but it remains to be seen whether this holds true in the nanosecond pulse regime. ${ }^{19,20}$

Gold nanorods are known to be unstable under laser irradiation and may undergo melting and reshaping when exposed to nanosecond pulsed irradiation. This effect coupled with the high bubble generation thresholds observed in this study suggests that nanorods may not be an ideal choice of particle for in vivo bubble generation. ${ }^{33}$ Increasing nanorod size showed a reduction in bubble threshold that roughly scaled inversely with the absorption cross section. The largest nanorod studied, with a length of $160 \mathrm{~nm}$ and width of $44 \mathrm{~nm}$, had a bubble generation threshold similar to that of the nanoshells, but still slightly above the MPE. Silica coating was observed to significantly reduce the bubble threshold and can also improve photostability due to steric confinement of the gold, even if melting occurs.

In summary, gold nanoshells appear to be the preferable particle shape for bubble generation due to their thresholds being near or below the MPE. Previous work demonstrating the robustness of nanoshells under off-resonance femtosecond pulsed irradiation suggests that it may be possible to generate bubbles without damaging the nanoshells, alleviating concerns about harmful bioeffects due to nanoparticle melting and evaporation. Preliminary results from our group indicate that the damage threshold for the 660- and 980-nm resonant shells is higher than their bubble generation threshold, while the 800-nm resonant shells undergo damage prior to reaching the bubble threshold. Further studies are necessary to elucidate the effects of nanoparticle agglomeration in vitro and in vivo on bubble generation thresholds.

\section{Conclusions}

We have developed and validated a multimodal testing platform for characterizing bubble generation thresholds of PNPs. Measurements of gold nanospheres, nanorods, and nanoshells indicated strong dependence of bubble generation thresholds on particle geometry and composition. Notably, some PNPs are capable of generating bubbles at exposure levels below ANSI safety limits for skin. The impact of potential bubble generation on safety and performance should thus be considered during development of PNPs and devices intended to be combined for diagnostic or therapeutic applications.

\section{Disclosures}

The authors have no relevant financial interests in this article and no potential conflicts of interest to disclose.

\section{Acknowledgments}

The authors acknowledge the FDA Advanced Characterization Facility for use of their TEM. This project was supported by the CDRH/FDA Nanotechnology Research Program. The project 
was supported in part by an appointment to the ORISE Research Participation Program at the Center for Devices and Radiological Health administered by the Oak Ridge Institute for Science and Education through an agreement between the U.S. Department of Energy and CDRH. The authors would also like to acknowledge constructive input from Dr. Pejhman Ghassemi (FDA) on in vitro approaches for evaluating spectral changes in nanoparticle absorption. Portions of this work were presented at SPIE Photonics West in 2018. ${ }^{39}$ Disclaimer: The mention of commercial products, their sources, or their use in connection with material reported herein is not to be construed as either an actual or implied endorsement of such products by the U.S. Department of Health and Human Services.

\section{References}

1. N. Khlebtsov et al., "Analytical and theranostic applications of gold nanoparticles and multifunctional nanocomposites," Theranostics $\mathbf{3}(3)$, 167-180 (2013).

2. A. M. Fales, B. M. Crawford, and T. Vo-Dinh, "Folate receptor-targeted theranostic nanoconstruct for surface-enhanced Raman scattering imaging and photodynamic therapy," ACS Omega 1(4), 730-735 (2016).

3. C. Andreou, S. A. Kishore, and M. F. Kircher, "Surface-enhanced Raman spectroscopy: a new modality for cancer imaging," J. Nucl. Med. 56(9), 1295-1299 (2015).

4. H. Arami et al., "Nanomedicine for spontaneous brain tumors: a companion clinical trial," ACS Nano 13(3), 2858-2869 (2019).

5. W. Li and X. Chen, "Gold nanoparticles for photoacoustic imaging," Nanomedicine 10(2), 299-320 (2015).

6. B. M. Crawford et al., "Photothermal ablation of inflammatory breast cancer tumor emboli using plasmonic gold nanostars," Int. J. Nanomed. 12, 6259-6272 (2017).

7. Z. Qin and J. C. Bischof, "Thermophysical and biological responses of gold nanoparticle laser heating," Chem. Soc. Rev. 41(3), 1191-1217 (2012).

8. E. Y. Lukianova-Hleb et al., "Intraoperative diagnostics and elimination of residual microtumours with plasmonic nanobubbles," Nat Nano 11(6), 525-532 (2016).

9. A. M. Wilson et al., "In vivo laser-mediated retinal ganglion cell optoporation using KV1.1 conjugated gold nanoparticles," Nano Lett. 18(11), 6981-6988 (2018).

10. S. Peeters et al., "Mechanisms of nanoparticle-mediated photomechanical cell damage," Biomed. Opt. Express 3(3), 435-446 (2012).

11. E. Lukianova-Hleb et al., "Plasmonic nanobubbles as transient vapor nanobubbles generated around plasmonic nanoparticles," ACS Nano 4(4), 2109-2123 (2010).

12. A. Siems et al., "Thermodynamics of nanosecond nanobubble formation at laser-excited metal nanoparticles," N. J. Phys. 13(4), 043018 (2011).

13. K. Metwally, S. Mensah, and G. Baffou, "Fluence threshold for photothermal bubble generation using plasmonic nanoparticles," J. Phys. Chem. C 119(51), 28586-28596 (2015).

14. J. Lombard, T. Biben, and S. Merabia, "Threshold for vapor nanobubble generation around plasmonic nanoparticles," J. Phys. Chem. C 121(28), 15402-15415 (2017).

15. American National Standards Institute, "American national standard for the safe use of lasers," in Z136.1, ANSI, Orlando, Florida (2014).

16. D. Lapotko, "Optical excitation and detection of vapor bubbles around plasmonic nanoparticles," Opt. Express 17(4), 2538-2556 (2009).

17. G. Akchurin et al., "Gold nanoshell photomodification under a singlenanosecond laser pulse accompanied by color-shifting and bubble formation phenomena," Nanotechnology 19(1), 015701 (2008).

18. L. Cavigli et al., "Light activated microbubbles for imaging and microsurgery," Proc. SPIE 10064, 1006457 (2017).

19. R. Lachaine et al., "Computational design of durable spherical nanoparticles with optimal material, shape, and size for ultrafast plasmonenhanced nanocavitation," ACS Photonics 3(11), 2158-2169 (2016).

20. R. Lachaine et al., "Rational design of plasmonic nanoparticles for enhanced cavitation and cell perforation," Nano Lett. 16(5), 3187-3194 (2016).
21. É. Boulais, R. Lachaine, and M. Meunier, "Plasma-mediated nanocavitation and photothermal effects in ultrafast laser irradiation of gold nanorods in water," J. Phys. Chem. C 117(18), 9386-9396 (2013).

22. M. O. Ogunyankin et al., "Optimizing the NIR fluence threshold for nanobubble generation by controlled synthesis of $10-40 \mathrm{~nm}$ hollow gold nanoshells," Adv. Funct. Mater. 28(10), 1705272 (2018).

23. S. Mallidi et al., "Multiwavelength photoacoustic imaging and plasmon resonance coupling of gold nanoparticles for selective detection of cancer," Nano Lett. 9(8), 2825-2831 (2009).

24. H. Moon et al., "Amplified photoacoustic performance and enhanced photothermal stability of reduced graphene oxide coated gold nanorods for sensitive photoacoustic imaging," ACS Nano 9(3), 2711-2719 (2015).

25. S. Liang et al., "CD44v6 monoclonal antibody-conjugated gold nanostars for targeted photoacoustic imaging and plasmonic photothermal therapy of gastric cancer stem-like cells," Theranostics 5(9), 970-984 (2015).

26. M. A. Yurkin and A. G. Hoekstra, "The discrete-dipole-approximation code ADDA: Capabilities and known limitations," J. Quant. Spectrosc. Radiat. Transfer 112(13), 2234-2247 (2011).

27. M. Kitz et al., "Vapor bubble generation around gold nano-particles and its application to damaging of cells," Biomed. Opt. Express 2(2), 291-304 (2011)

28. S. Wang et al., "Quantitative evaluation and optimization of photothermal bubble generation around overheated nanoparticles excited by pulsed lasers," J. Phys. Chem. C 122(42), 24421-24435 (2018).

29. A. M. Fales et al., "Quantitative evaluation of nanosecond pulsed laserinduced photomodification of plasmonic gold nanoparticles," Sci. Rep. 7(1), 15704 (2017).

30. A. M. Fales et al., "Pulsed laser damage of gold nanorods in turbid media and its impact on multi-spectral photoacoustic imaging," Biomed. Opt. Express 10(4), 1919-1934 (2019).

31. R. Huschka et al., "Light-induced release of DNA from gold nanoparticles: nanoshells and nanorods," J. Am. Chem. Soc. 133(31), 12247-12255 (2011).

32. U. Constantin et al., "The 'nanobig rod' class of gold nanorods: optimized dimensions for improved in vivo therapeutic and imaging efficacy," Nanotechnology 24(21), 215102 (2013).

33. L. Cavigli et al., "Size affects the stability of the photoacoustic conversion of gold nanorods," J. Phys. Chem. C 118(29), 16140-16146 (2014).

34. Y.-S. Chen et al., "Silica-coated gold nanorods as photoacoustic signal nanoamplifiers," Nano Lett. 11(2), 348-354 (2011).

35. V. K. Pustovalov et al., "Thermo-optical analysis and selection of the properties of absorbing nanoparticles for laser applications in cancer nanotechnology," Cancer Nanotechnol. 1(1), 35-46 (2010).

36. G. A. Pang et al., "Theoretical and experimental study of photoacoustic excitation of silica-coated gold nanospheres in water," arXiv:1905. 06227 (2019)

37. A. Doppenberg, M. Meunier, and C. Boutopoulos, "A needle-like optofluidic probe enables targeted intracellular delivery by confining light-nanoparticle interaction on single cell," Nanoscale 10(46), 21871-21878 (2018).

38. L. Poon et al., "Photothermal release of single-stranded DNA from the surface of gold nanoparticles through controlled denaturating and Au-S bond breaking," ACS Nano 4(11), 6395-6403 (2010).

39. A. M. Fales et al., "Size-dependent thresholds for melting and nanobubble generation using pulsed-laser irradiated gold nanoparticles," Proc. SPIE 10509, 105090C (2018).

Andrew M. Fales received his BS degree in biochemistry and molecular biology from the University of Maryland, Baltimore County, in 2010, and his $\mathrm{PhD}$ in biomedical engineering from Duke University, North Carolina, in 2016. Since then, he has been conducting research at the FDA, studying the interactions between pulsed laser light and plasmonic nanoparticles. His current research interests include plasmonics, pulsed laser-nanoparticle interactions, and Raman spectroscopy.

William C. Vogt received his BS degree in mechanical engineering from the University of Massachusetts Amherst in 2009 and his PhD in biomedical engineering from Virginia Polytechnic Institute and State University in 2013. Since 2013, he has been conducting photoacoustic imaging research at the FDA in the Office of Science and Engineering Laboratories. His research interests include 
photoacoustic imaging, phantom-based image quality testing, and biophotonic medical device characterization and evaluation.

Keith A. Wear received his MS degree and PhD in applied physics from Stanford University, California, in 1982 and 1987, respectively. Since 1989, he has worked at the FDA. He is an associate editor of three journals: Journal of the Acoustical Society of America; IEEE Transactions on Ultrasonics, Ferroelectrics, and Frequency Control, and Ultrasonic Imaging. He is a fellow of the Acoustical Society of America, the American Institute for Medical and Biological Engineering, and the American Institute of Ultrasound in Medicine.

T. Joshua Pfefer received his $\mathrm{PhD}$ in biomedical engineering from the University of Texas at Austin and was a research fellow at the Wellman Laboratories of Photomedicine. In 2000, he joined the FDA, where he is currently the leader of the Optical Diagnostic Devices Laboratory. His group's research focuses on safety and effectiveness in emerging clinical biophotonic spectroscopy and imaging technologies. He is a fellow of SPIE.

Ilko K. Ilev is the leader of Optical Therapeutics and Medical Nanophotonics Laboratory, and a Senior Biomedical Research Service Scientist at FDA. He has a PhD in quantum and laser physics. $\mathrm{He}$ has over 25 years of experience in the United States, Europe, and Japan, with more than 415 publications in the field of laser technologies, biophotonics, nanobiophotonics, bioimaging, biosensing, and laser safety. He is an elected fellow of IEEE, OSA, SPIE, and ASLMS. 